\title{
Potential etiology, prevalence of cirrhosis, and mode of detection among patients with non-B non-C hepatocellular carcinoma in Korea
}

Jihye Kim, Wonseok Kang, Dong Hyun Sinn, Geum-Youn Gwak, Yong-Han Paik, Moon Seok Choi, Joon Hyeok Lee, Kwang Cheol Koh, and Seung Woon Paik

Department of Medicine, Samsung Medical Center, Sungkyunkwan University School of Medicine, Seoul, Korea

Received: January 28, 2018

Revised : May 16, 2018

Accepted: May 28, 2018

\section{Correspondence to}

Seung Woon Paik, M.D.

Department of Medicine,

Samsung Medical Center,

Sungkyunkwan University

School of Medicine, 81 Irwon-ro,

Gangnam-gu, Seoul 06351, Korea

Tel: +82-2-3410-3409

Fax: +82-2-3410-6983

E-mail: sw.paik@samsung.com
Background/Aims: We systematically evaluated the clinical characteristics, prevalence of cirrhosis, and mode of detection in virus-unrelated (non-B non-C, NBNC) hepatocellular carcinoma (HCC) patients in Korea.

Methods: A total of 447 consecutive treatment-naïve NBNC-HCC adult patients who were registered at the Samsung Medical Center HCC registry in Korea from 2010 to 2013 were analyzed. NBNC was defined as negative hepatitis B surface antigen and negative anti-hepatitis $\mathrm{C}$ virus antibody. Presence of cirrhosis was determined based on histological, radiological, endoscopic, and serologic results. Mode of detection was classified as either under surveillance, incidental, or symptomatic.

Results: Heavy alcohol use was the most common potential etiology in NBNCHCC (NBNC-A, alcohol) (59.7\%). Ten patients had other identifiable causes (NBNC-O, other identifiable cause) such as autoimmune hepatitis. The rest (38.0\%) had no-identifiable cause (NBNC-NA-NO, non-alcohol, no-other identifiable cause). In NBNC-NA-NO group, 83.5\% (96/115) of patients with available hepatitis B core immunoglobulin G antibody ( $\mathrm{HBcIgG}$ ) showed HBcIgG positivity, and $80.6 \%(137 / 170)$ had metabolic risk factors (diabetes, obesity, hypertension, and/ or dyslipidemia). Cirrhosis was present in $90.0 \%, 70.4 \%$, and $60.0 \%$ of NBNC-O, NBNC-A, and NBNC-NA-NO patients, respectively. The proportion of patients diagnosed under surveillance was $25.5 \%$ across all patients, with specific proportions being $80.0 \%, 27.7 \%$, and $18.8 \%$ for NBNC-O, NBNC-A, and NBNC-NA-NO, respectively.

Conclusions: Among NBNC-HCC patients, heavy alcohol use or any other identifiable cause was not found in 38.0\%. These NBNC-NA-NO HCC patients showed a high prevalence of $\mathrm{HBcIgG}$ positivity and metabolic risk factors, suggesting that prior hepatitis B virus infection and metabolic risk factors may be major contributing factors in the hepatocarcinogenesis in NBNC-NA-NO patients.

Keywords: Non-B non-C; Carcinoma, hepatocellular; Etiology; Liver cirrhosis 


\section{INTRODUCTION}

Liver cancer is a leading cause of mortality in Korea as well as worldwide [1]. Hepatocellular carcinoma (HCC), the predominant form of liver cancer [2], usually develops in patients with chronic liver disease. Hepatitis B virus (HBV) and hepatitis $\mathrm{C}$ virus (HCV) are the most common etiologies of HCC worldwide. However, a nationwide cohort study has shown that non-B non-C (NBNC) HCC constitutes about one-fourth of the cases of HCC in Korea [3]. Potential etiologies for NBNC HCC include heavy alcohol use, autoimmune liver disease, vascular liver disease, genetic liver disease, non-alcoholic fatty liver disease (NAFLD), or prior HBV exposure.

In a study from a tertiary hospital in Korea, which assessed 329 NBNC HCC cases without heavy alcohol intake diagnosed between 2001 and 2010, prior HBV infection still showed a greater etiological role than that of NAFLD [4]. In another study from a tertiary hospital in Korea, which assessed 523 NBNC HCC cases diagnosed between 2007 and 2009, 47.4\% of NBNC HCC cases (not including those with specific causes) were associated with prior HBV exposure, while $10.6 \%$ and $2.8 \%$ had chronic alcohol intake or metabolic syndrome alone, respectively, as a risk factor, and many (39.2\%) had two or three risk factors as a potential etiology [5]. In another study that reported the clinical characteristics of 480 NBNC HCC patients diagnosed between 2003 and 2012 in a tertiary hospital in Korea, NBNC-HCC was reported to be associated with older age at diagnosis, more frequent occurrence of metabolic syndrome, and less aggressive tumor characteristics [6], and burnt-out NAFLD has been suggested as a major cause of cryptogenic HCC.

NAFLD is a chronic liver disease, characterized by the findings of fat infiltration of the liver without significant alcohol intake or secondary causes for hepatic steatosis [7]. NAFLD can progress to end-stage liver disease, such as cirrhosis and HCC. The incidence of obesity-related metabolic syndrome is rapidly increasing in Korea, resulting in an increasing prevalence of NAFLD [7]. In contrast, the prevalence of HBV has recently been decreasing, especially among the younger generation [8]. Hence, the relative etiological role of prior HBV infection and NAFLD in the development of NBNC HCC is expected to change in Korea.
In this study, we investigated the clinical features of NBNC HCC patients with particular focus on the potential etiology, mode of detection and prevalence of cirrhosis, using a more recent cohort (NBNC HCC patients diagnosed between 2010 and 2013), to investigate the clinical features of NBNC HCC patients in Korea

\section{METHODS}

\section{Study setting and participants}

This study is a single-center, retrospective cohort study using the Samsung Medical Center HCC registry data. The HCC registry of Samsung Medical Center is a prospective registry that records baseline clinical characteristics, tumor variables, and the initial treatment modalities of every newly-diagnosed HCC patient aged 18 years or older who received care at the Samsung Medical Center. A diagnosis of HCC was established either histologically or clinically according to the regional HCC guideline $[9,10]$. We screened a total of 3,347 patients registered in the Samsung Medical Center HCC registry between January 2010 and December 2013. Among them, we included a total of 447 consecutive NBNC HCC patients, excluding those who had positive results for hepatitis B surface antigen (HBsAg) and/or anti-HCV. The study was approved by the Ethics Committee of Samsung Medical Center and was conducted in accordance with the principles of the Declaration of Helsinki. Because this study was based on a retrospective analysis of existing administrative and clinical data, the requirement for informed patient consent was waived by the Institutional Review Board of Samsung Medical Center (IRB No. 2018-01-151).

\section{Variables and data collection}

We used the following variables collected from the HCC registry: age at diagnosis, gender, body mass index (BMI), mode of detection, Child-Pugh Class, tumor characteristics (e.g., number of tumors, maximal tumor size, presence of vascular invasion and/or extrahepatic spread), serum alpha-fetoprotein (AFP), prothrombin-induced by vitamin $\mathrm{K}$ antagonist-II (PIVKA-II) levels and initial treatment modality. For this study, we additionally collected several variables, including the presence of autoimmune liver disease, vascular liver disease, genetic 
liver disease, history of heavy alcohol use, family history of HCC, smoking use, diabetes, hypertension, dyslipidemia, coronary arterial disease and hepatitis B core antibody results, liver imaging and endoscopy results by reviewing electronic medical records, as the HCC registry did not contain these variables. Overall survival was defined as the time from diagnosis to the last follow-up or to death, whichever came first. The referral date was September 30, 2017.

\section{Definitions}

In this study, we defined cirrhosis based on a combination of histology, imaging, endoscopy, and fibrosis-4 score (FIB-4). For those without histologic data, we defined cirrhosis by applying the following criteria in stepwise order: imaging based on nodular liver surface or caudate lobe hypertrophy, presence of ascites with/ without diuretic use, endoscopy showing varices, and FIB-4 score. The FIB-4 score was calculated as originally described, using age, serum aspartate aminotransferase, alanine aminotransferase, and platelet count [11]. We used a cutoff point of 3.25, which had a sensitivity of $55 \%$, a specificity of $97 \%$, and a positive predictive value of $65 \%$ for cirrhosis $[11,12]$.

The mode of detection was prospectively collected from the HCC registry by trained abstractors. The variable had three categories including under surveillance, incidental and symptomatic. In order to identify potential causes, the presence of specific liver disease, alcohol consumption, hepatitis B core IgG antibody, and metabolic diseases (diabetes, obesity, hypertension, and dyslipidemia) were examined. Heavy alcohol intake was defined as $>14$ standard drinks/week in males and $>7$ standard drinks in females or people aged $\geq 65$ years (14 $\mathrm{g}$ of alcohol per one standard drink) over a period of three years [13-15]. Those with identifiable chronic liver disease, such as autoimmune liver disease, vascular liver disease, and genetic liver disease were classified as NBNC-O (other identifiable causes). In the absence of other identifiable causes, patients were first categorized into NBNC-A (alcohol) if they had a history of heavy alcohol use. Patients without other identifiable causes or a history of heavy alcohol use were classified as NBNCNA-NO (non-alcohol, no-other identifiable causes). We reviewed the records of NBNC-NA-NO patients for prior HBV infection, defined by positivity for hepatitis B core immunoglobulin G antibody (HBcIgG), and looked for the presence of metabolic risk factors, such as obesity $\left(\mathrm{BMI} \geq 25 \mathrm{~kg} / \mathrm{m}^{2}\right)$, diabetes, hypertension and/or dyslipidemia. Diabetes was defined according to the American Diabetes Association guideline as follows: hemoglobin Alc $\geq 6.5 \%$, fasting plasma glucose $\geq 126 \mathrm{mg} / \mathrm{dL}$, 2-hour plasma glucose $\geq 200 \mathrm{mg} / \mathrm{dL}$ during an oral glucose tolerance test, or classic symptoms with random plasma glucose $\geq 200 \mathrm{mg} / \mathrm{dL}$ [16]. Hypertension was defined as $\geq$ $130 / 85 \mathrm{mmHg}$ or in the current use of anti-hypertensive medication. Dyslipidemia was defined as total cholesterol $\geq 200 \mathrm{mg} / \mathrm{dL}$ or current statin use.

\section{Statistical analysis}

All statistical analyses were conducted using SPSS version 24 software package (IBM Co., Armonk, NY, USA). Data are shown as the median (range), median (interquartile range), or number (\%) of patients. The chisquare test, Fisher's exact test, and Mann-Whitney test were used to compare data regarding the baseline characteristics and variables between subgroups. Survival analyses were performed using the Kaplan-Meier method with the differences in survival curves assessed using the log-rank test. Cox proportional hazards models were used to estimate the hazard ratios for the variables. For all analyses, two-sided tests of significance were used with a $p$ value of less than 0.05 considered to be statistically significant.

\section{RESULTS}

\section{Baseline characteristics and overall survival}

The baseline characteristics of the study patients are summarized in Table 1. The median age of patients was 67 years. In terms of age distribution, $77.0 \%$ of patients were aged above 60 years. A majority of the patients (85.2\%) were males. Most had preserved liver function, indicated by Child-Pugh class A (87.0\%, 389/447). The number of tumors was single in $64.4 \%$ of patients. Portal vein invasion was observed in $20.1 \%$ of patients (90/447). The most common initial treatment modality was transarterial chemoembolization, followed by surgical resection. Mortality was observed in 209 patients during the median follow-up of 32.2 months (range, 0.1 to 90.3). The 1-, 3-, and 5-year survival rates were $72.7 \%$, 
Table 1. Overall characteristics of study population $(n=447)$

\begin{tabular}{|c|c|}
\hline Variable & Value \\
\hline Age, yr & $67(26-88)$ \\
\hline$<50$ & $29(6.5)$ \\
\hline $50-59$ & $74(16.6)$ \\
\hline $60-69$ & $167(37.4)$ \\
\hline$\geq 70$ & $177(39 \cdot 6)$ \\
\hline Male sex & $381(85.2)$ \\
\hline Body mass index ${ }^{\mathrm{a}}, \mathrm{kg} / \mathrm{m}^{2}$ & $24.5(15.9-37.6)$ \\
\hline$<18.5$ & $10(2.2)$ \\
\hline $18.5-22.9$ & $143(32.0)$ \\
\hline $23.0-24.9$ & $95(21.3)$ \\
\hline$\geq 25$ & $191(42.7)$ \\
\hline Family history of HCC & $29(6.5)$ \\
\hline Heavy alcohol use & $269(60.2)$ \\
\hline \multicolumn{2}{|l|}{ Smoking } \\
\hline Never & $200(44.7)$ \\
\hline Ex-smoker & $133(29.8)$ \\
\hline Current smoker & $114(25 \cdot 5)$ \\
\hline Diabetes & $211(47.2)$ \\
\hline Hypertension & $246(55.0)$ \\
\hline Dyslipidemia & $46(10.3)$ \\
\hline Coronary arterial disease & $25(5.8)$ \\
\hline \multicolumn{2}{|l|}{ Mode of detection } \\
\hline Under surveillance & $114(25 \cdot 5)$ \\
\hline Incidental & $196(43.8)$ \\
\hline Symptomatic & $137(30.6)$ \\
\hline \multicolumn{2}{|l|}{$\mathrm{HBcIgG}^{\mathrm{b}}$} \\
\hline Yes & $235(52.6)$ \\
\hline No & $58(13.0)$ \\
\hline \multicolumn{2}{|l|}{ Child-Pugh class } \\
\hline A & $389(87.0)$ \\
\hline $\mathrm{B}$ & $53(11.9)$ \\
\hline $\mathrm{C}$ & $5(1.1)$ \\
\hline Albumin, g/dL & $4.2(2.4-5 \cdot 3)$ \\
\hline Bilirubin, g/dL & $0.8(0.2-37.8)$ \\
\hline
\end{tabular}

$47.4 \%$, and $16.3 \%$, respectively. In unadjusted analysis, the mode of detection, presence of liver cirrhosis, Child-Pugh class, Eastern Cooperative Oncology Group (ECOG) performance, number of tumors, tumor size, portal vein thrombosis, AFP levels, and initial treatment

\begin{tabular}{|c|c|}
\hline Variable & Value \\
\hline $\mathrm{AST}, \mathrm{U} / \mathrm{L}$ & $39(13-659)$ \\
\hline ALT, U/L & $27(7-368)$ \\
\hline PT, INR & $1.05(0.85-6.19)$ \\
\hline Platelet, $\times 10^{3} / \mu \mathrm{L}$ & $161(17-544)$ \\
\hline \multicolumn{2}{|l|}{ No. of tumors } \\
\hline 1 & $288(64.4)$ \\
\hline $2-3$ & $98(22.0)$ \\
\hline$\geq 4$ & $41(9.1)$ \\
\hline Diffuse or infiltrative & $20(4 \cdot 5)$ \\
\hline \multicolumn{2}{|c|}{ Maximal tumor diameter, $\mathrm{cm}$} \\
\hline$<5$ & $250(55 \cdot 9)$ \\
\hline$\geq 5$ & $197(44.1)$ \\
\hline Portal vein invasion & $90(20.1)$ \\
\hline $\mathrm{AFP}^{\mathrm{c}}$ & $11.1(0.9-600,000)$ \\
\hline$<10$ & $207(46.3)$ \\
\hline $10-100$ & $105(23.5)$ \\
\hline$\geq 100$ & $134(30.0)$ \\
\hline PIVKA-II & $120(6-75,000)$ \\
\hline \multicolumn{2}{|l|}{ Initial treatment modality } \\
\hline Resection & $117(26.2)$ \\
\hline RF ablation & $72(16.1)$ \\
\hline TACE & $177(39 \cdot 6)$ \\
\hline Others & $39(8.7)$ \\
\hline Best supportive care & $42(9.4)$ \\
\hline
\end{tabular}

Values are presented as median (range) or number (\%).

HCC, hepatocellular carcinoma; HBcIgG, hepatitis B core immunoglobulin G antibody; AST, aspartate aminotransferase; ALT, alanine aminotransferase; PT, prothrombin time; INR, international normalized ratio; AFP, alpha-fetoprotein; PIVKA-II, prothrombin-induced by vitamin K antagonist-II; RF, radiofrequency; TACE, transarterial chemoembolization.

${ }^{\mathrm{a}}$ Missing in eight patients.

${ }^{\mathrm{b}}$ Missing in 154 patients.

${ }^{\mathrm{c}}$ Missing in one patient.

modality were all associated with survival (Table 2). In multivariable adjusted analysis, tumor size, portal vein thrombosis, and initial treatment modality were found to be independent factors for survival (Table 2). 
Kim J, et al. Non-B non-C HCC

Table 2. Risk factors for overall survival

\begin{tabular}{|c|c|c|c|c|}
\hline \multirow{2}{*}{ Variable } & \multicolumn{2}{|c|}{ Unadjusted } & \multicolumn{2}{|c|}{ Multivariable } \\
\hline & $\operatorname{HR}(95 \% \mathrm{CI})$ & $p$ value & $\operatorname{HR}(95 \% \mathrm{CI})$ & $p$ value \\
\hline Age & $1.00(0.99-1.01)$ & 0.989 & $1.00(0.99-1.02)$ & 0.680 \\
\hline Male sex & $0.83(0.57-1.19)$ & 0.305 & & \\
\hline Body mass index & $1.00(0.96-1.04)$ & 0.810 & & \\
\hline Heavy alcohol use & $1.21(0.91-1.61)$ & 0.194 & $1.20(0.89-1.63)$ & 0.237 \\
\hline Diabetes & $0.86(0.66-1.13)$ & 0.284 & & \\
\hline Hypertension & $0.87(0.66-1.14)$ & 0.297 & & \\
\hline Dyslipidemia & $1.06(0.67-1.66)$ & 0.816 & & \\
\hline Family history of HCC & $0.92(0.52-1.61)$ & 0.768 & & \\
\hline \multicolumn{5}{|l|}{ Smoking } \\
\hline Never & 1 & & & \\
\hline Ex-smoker & $0.98(0.71-1.33)$ & 0.872 & & \\
\hline Current smoker & $0.81(0.57-1.15)$ & 0.231 & & \\
\hline \multicolumn{5}{|l|}{ Mode of detection } \\
\hline Under surveillance & 1 & & 1 & \\
\hline Incidental & $0.82(0.57-1.18)$ & 0.286 & $0.81(0.55-1.19)$ & 0.282 \\
\hline Symptomatic & $2.12(1.50-2.99)$ & $<0.001$ & $1.24(0.82-1.87)$ & 0.315 \\
\hline \multicolumn{5}{|l|}{ HBcIgG } \\
\hline No & 1 & & 1 & \\
\hline Yes & $0.80(0.54-1.18)$ & 0.263 & $0.76(0.50-1.15)$ & 0.191 \\
\hline Missing & $0.68\left(0.45^{-1.03}\right)$ & 0.068 & $0.78(0.50-1.21)$ & 0.273 \\
\hline Liver cirrhosis & $1.56(1.14-2.13)$ & 0.005 & $1.25(0.88-1.77)$ & 0.211 \\
\hline \multicolumn{5}{|l|}{ Child-Pugh class } \\
\hline A & 1 & & 1 & \\
\hline $\mathrm{B}$ & $2.11(1.47-3.02)$ & $<0.001$ & $1.28(0.83-1.98)$ & 0.264 \\
\hline $\mathrm{C}$ & $5.74(2.34-14.09)$ & $<0.001$ & $1.90(0.69-5.22)$ & 0.215 \\
\hline \multicolumn{5}{|l|}{ ECOG } \\
\hline o & 1 & & 1 & \\
\hline$\geq 1$ & $1.50(1.01-2.24)$ & 0.045 & $1.01(0.65-1.58)$ & 0.961 \\
\hline \multicolumn{5}{|l|}{ No. of tumors } \\
\hline Single & 1 & & 1 & \\
\hline Multiple & $1.79(1.34-2.38)$ & $<0.001$ & $1.14(0.83-1.56)$ & 0.430 \\
\hline Diffuse/infiltrative & $8.14(4.96-13.38)$ & $<0.001$ & $1.58(0.88-2.86)$ & 0.126 \\
\hline \multicolumn{5}{|l|}{ Tumor size, $\mathrm{cm}$} \\
\hline$<5$ & 1 & & 1 & \\
\hline$\geq 5$ & $2.70(2.05-3.56)$ & $<0.001$ & $1.76(1.19-2.60)$ & 0.005 \\
\hline Portal vein thrombosis & $4.23(3.16-5.66)$ & $<0.001$ & $2.10(1.44-3.06)$ & $<0.001$ \\
\hline $\log _{10}$ AFP & $1.40(1.28-1.54)$ & $<0.001$ & $1.06(0.94-1.20)$ & 0.333 \\
\hline \multicolumn{5}{|c|}{ Initial treatment modality } \\
\hline Resection & 1 & & 1 & \\
\hline RF ablation & $1.73(0.90-3.33)$ & 0.100 & $2.25(1.12-4.54)$ & 0.023 \\
\hline TACE & $5.59(3.39-9.25)$ & $<0.001$ & $4.09(2.40-6.98)$ & $<0.001$ \\
\hline Others & $10.19(5.61-18.51)$ & $<0.001$ & $6.02(3.22-11.26)$ & $<0.001$ \\
\hline Best supportive care & $43.25(23.99-77.98)$ & $<0.001$ & $24.09(12.29-47.22)$ & $<0.001$ \\
\hline
\end{tabular}

HR, hazard ratio; CI, confidence interval; HCC, hepatocellular carcinoma; HBcIgG, hepatitis B core immunoglobulin G antibody; ECOG, Eastern Cooperative Oncology Group; AFP, alpha-fetoprotein; RF, radiofrequency; TACE, transarterial chemoembolization. 


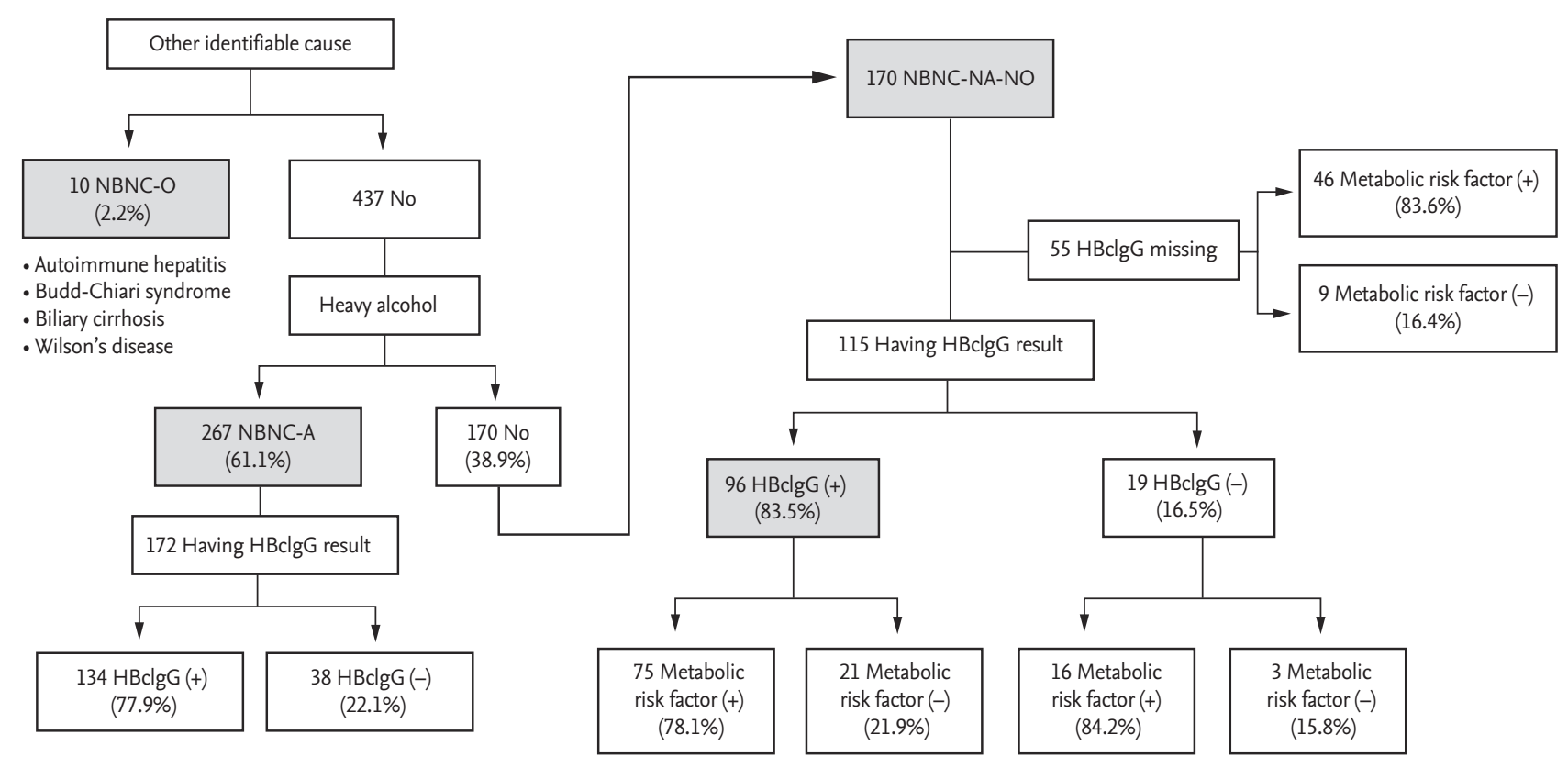

Figure 1. Potential etiology of non-B non-C (NBNC) hepatocellular carcinoma. NBNC-O, other identifiable cause; NBNC-A, alcohol; NBNC-NA-NO, non-alcohol, no-other identifiable cause; HBcIgG, hepatitis B core immunoglobulin G antibody.

\section{Potential etiology and patient profiles based on potential etiology}

The potential etiologies of NBNC-HCC are presented in Fig. 1. Ten patients had specific identifiable causes (NBNC-O); four patients had autoimmune hepatitis, two had Budd-Chiari syndrome, three had biliary cirrhosis, and one had Wilson's disease. Among 437 patients without other identifiable causes, 61.1\% (267/437) had a history of heavy alcohol use (NBNC-A). Of the 170 patients without heavy alcohol use or other identifiable causes (NBNC-NA-NO), 115 patients (67.6\%) had available HBcIgG test results with $83.5 \%$ of them (96/115) being positive for the antibody. Metabolic risk factors, specifically diabetes, obesity, hypertension, and dyslipidemia were detected in $49.4 \%, 42.9 \%, 61.2 \%$, and $14.7 \%$ of the patients, respectively.

Patients characteristics were compared according to potential etiology (NBNC-A vs. NBNC-NA-NO) as shown in Table 3. NBNC-NA-NO patients were characterized by an older age at diagnosis (about half were diagnosed at an age above 70 years), while the NBNC-A group was almost exclusively composed of male patients (99.3\%). Compared with NBNC-A patients, the group of NBNC-NA-NO patients was older and majority female with a greater incidence of dyslipidemia, family history of HCC, lower prevalence of liver cirrhosis, higher prevalence of single tumor and more favorable Barcelona Clinic Liver Cancer (BCLC) stage at diagnosis. Additionally, a higher number of NBNC-NA-NO patients underwent tumor resection. The presence of obesity or diabetes, HBcIgG positivity, Child-Pugh class, tumor size, incidence of portal vein thrombosis, and tumor markers did not significantly differ between the two groups. The incidence of hypertension was higher (61.2\% vs. 52.1\%, $p$ $=0.061)$ and the proportion of cases detected under surveillance was lower (18.8\% vs. $27.7 \%, p=0.090$ ) in NBNCNA-NO patients than in NBNC-A patients, although this difference was statistically marginal. There was no significant difference in overall survival between NBNCNA-NO and NBNC-A patients $(p=0.162)$ (Fig. 2).

When NBNC-NA-NO patients were compared according to $\mathrm{HBcIgG}$ positivity, there was no significant difference found in their baseline characteristics according to $\mathrm{HBcIgG}$ positivity, except that patients without $\mathrm{HBcIgG}$ presented with symptom more frequently (Supplementary Table 1). However, only 19 patients were HBcIgG negative. 
Kim J, et al. Non-B non-C HCC

Table 3. Comparison of NBNC-A versus NBNC-NA-NO ( $n=437)$

\begin{tabular}{|c|c|c|c|}
\hline Variable & NBNC-A $(n=267)$ & NBNC-NA-NO $(\mathrm{n}=170)$ & $p$ value \\
\hline Age, yr & & & 0.011 \\
\hline$<50$ & $16(6.0)$ & $11(6.4)$ & \\
\hline $50-59$ & $52(19.5)$ & $22(12.9)$ & \\
\hline $60-69$ & $110(41.2)$ & $55(32.4)$ & \\
\hline$\geq 70$ & $89(33.3)$ & $82(48.2)$ & \\
\hline Male sex & $265(99 \cdot 3)$ & $112(65.9)$ & $<0.001$ \\
\hline Body mass index ${ }^{\mathrm{a}}, \mathrm{kg} / \mathrm{m}^{2}$ & & & 0.994 \\
\hline$<18.5$ & $6(2.2)$ & $4(2.4)$ & \\
\hline $18.5-22.9$ & $85(31.8)$ & $56(32.9)$ & \\
\hline $23.0-24.9$ & $58(21.7)$ & $34(20.0)$ & \\
\hline$\geq 25$ & $113(42.3)$ & $73(42.9)$ & \\
\hline Diabetes & $122(45.7)$ & $84(49.4)$ & 0.448 \\
\hline Hypertension & $139(52.1)$ & $104(61.2)$ & 0.061 \\
\hline Dyslipidemia & $20(7.5)$ & $25(14.7)$ & 0.023 \\
\hline Family history of HCC & $9(3.4)$ & $20(11.8)$ & 0.001 \\
\hline Mode of detection & & & 0.090 \\
\hline Under surveillance & $74(27.7)$ & $32(18.8)$ & \\
\hline Incidental & $111(41.6)$ & $84(49.4)$ & \\
\hline Symptomatic & $82(30.7)$ & $54(31.8)$ & \\
\hline $\mathrm{HBcIgG}^{\mathrm{b}}$ & & & 0.399 \\
\hline Yes & $134(50.2)$ & $96(56.5)$ & \\
\hline No & $38(14.2)$ & $19(11.2)$ & \\
\hline Liver cirrhosis & $188(70.4)$ & $102(60.0)$ & 0.029 \\
\hline Child-Pugh class & & & 0.657 \\
\hline A & $229(85.8)$ & $151(88.8)$ & \\
\hline B & $35(13.1)$ & $17(10.0)$ & \\
\hline $\mathrm{C}$ & $3(1.1)$ & $2(1.2)$ & \\
\hline No. of tumors & & & 0.003 \\
\hline Single & $155(58.1)$ & $125(73.5)$ & \\
\hline Multiple & $99(37.1)$ & $38(22.4)$ & \\
\hline Diffuse/infiltrative & $13(4.9)$ & $7(4.1)$ & \\
\hline Tumor size, $\mathrm{cm}$ & & & 0.168 \\
\hline$<5$ & $155(58.1)$ & $87(51.2)$ & \\
\hline$\geq 5$ & $112(41.9)$ & $83(48.8)$ & \\
\hline Portal vein thrombosis & $54(20.2)$ & $34(20.0)$ & 0.954 \\
\hline BCLC stage & & & 0.042 \\
\hline o & $24(9.0)$ & $18(10.6)$ & \\
\hline A & $105(39 \cdot 3)$ & $84(49.4)$ & \\
\hline B & $44(16.5)$ & $13(7.6)$ & \\
\hline $\mathrm{C}$ & $90(33.7)$ & $51(30.0)$ & \\
\hline $\mathrm{D}$ & $4(1.5)$ & $4(2.4)$ & \\
\hline AFP & $11.1(5.5-146.9)$ & $11.3(4.8-666.3)$ & 0.807 \\
\hline
\end{tabular}


Table 3. Continued

\begin{tabular}{|c|c|c|c|}
\hline Variable & NBNC-A $(n=267)$ & NBNC-NA-NO $(\mathrm{n}=170)$ & $p$ value \\
\hline PIVKA-II & $132.0(34.5-1,200.0)$ & $119.0(25.0-1,200.0)$ & 0.341 \\
\hline Initial treatment modality & & & 0.013 \\
\hline Resection & $59(21.9)$ & $58(32.6)$ & \\
\hline RF ablation & $40(14.9)$ & $32(18.0)$ & \\
\hline TACE & $121(45 \cdot 0)$ & $56(31.5)$ & \\
\hline Others & $27(10.0)$ & $12(6.7)$ & \\
\hline Best supportive care & $22(8.2)$ & $20(11.2)$ & \\
\hline
\end{tabular}

Values are presented as number (\%) or median (interquartile range).

NBNC-A, non-B non-C alcohol; NBNC-NA-NO, non-alcohol, no-other identifiable cause; HCC, hepatocellular carcinoma; HBcIgG, hepatitis B core immunoglobulin G antibody; BCLC, Barcelona Clinic Liver Cancer; AFP, alpha-fetoprotein; PIVKA-II, prothrombin-induced by vitamin K antagonist-II; RF, radiofrequency; TACE, transarterial chemoembolization.

${ }^{a}$ Missing in five and three patients, respectively.

${ }^{\mathrm{b}}$ Missing in $95(35.6 \%)$ and $55(32.4 \%)$ patients, respectively.

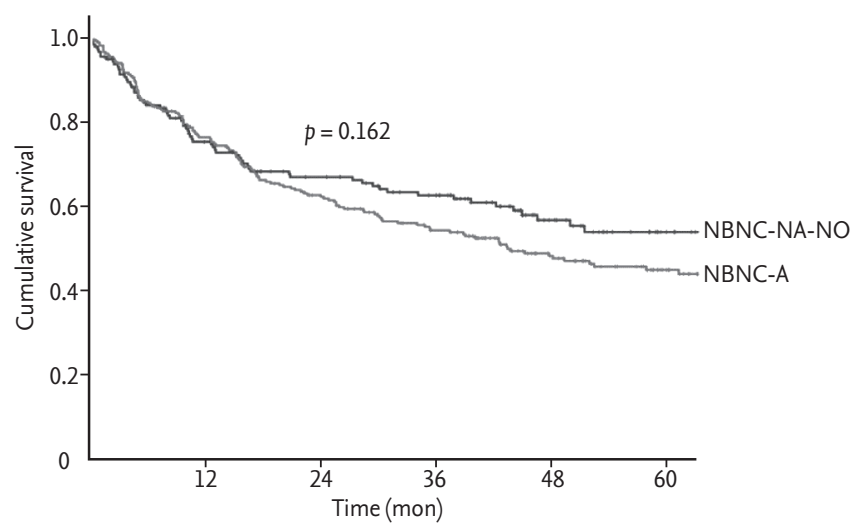

Figure 2. Kaplan-Meier curves for overall survival between non-B non-C (NBNC)-A and NBNC-NA-NO. NBNC-A, alcohol; NBNC-NA-NO, non-alcohol, no-other identifiable cause.

\section{Prevalence of cirrhosis and mode of detection}

Fig. 3 is a schematic diagram of the prevalence of cirrhosis based on histopathologic findings, imaging studies, the presence of varices on endoscopy, and an FIB-4 score greater than 3.25 as a cutoff point, sequentially. Overall, no evidence of cirrhosis was found histologically, radiologically, endoscopically, or through FIB-4 score in up to one-third $(33 \%, 148 / 447)$ of NBNC HCC patients. The prevalence of cirrhosis according to potential etiologies of HCC was the highest (90\%) in NBNC-O, followed by NBNC-A (70.4\%) and NBNC-NA-NO (60\%). The group of patients with cirrhosis was more comprised of patients with heavy alcohol use and diabetes, and less comprised

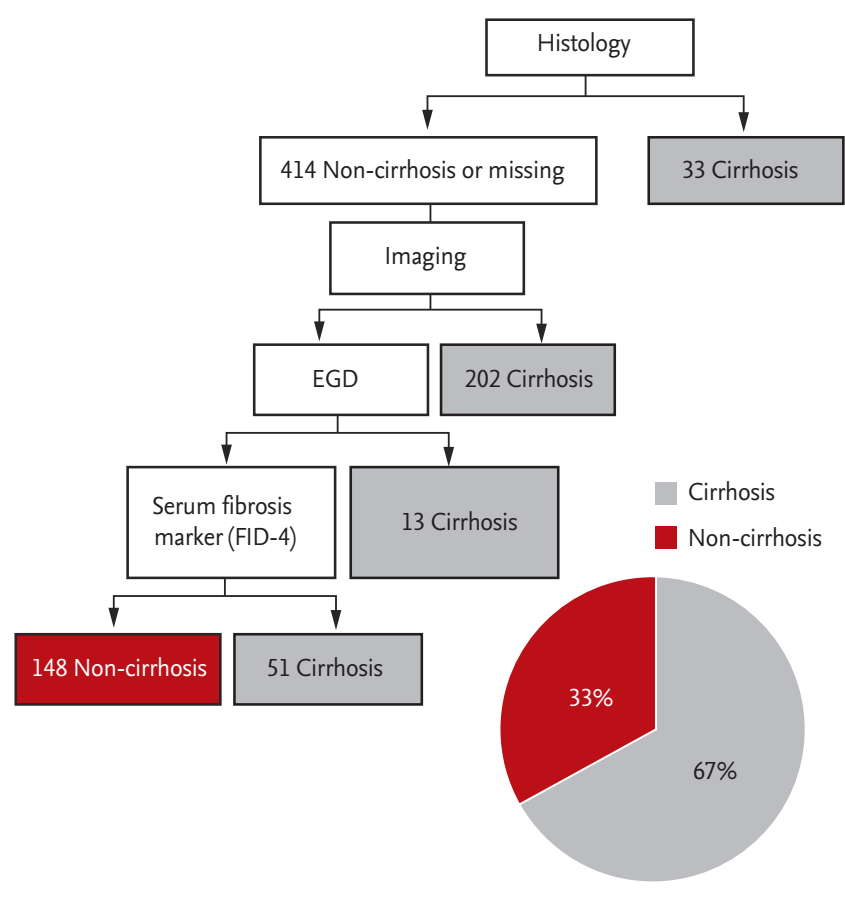

Figure 3. Prevalence of cirrhosis based on multiple criteria. EGD, esophagogastroduodenoscopy; FIB-4, fibrosis-4 score.

of patients with dyslipidemia, than the group of patients without cirrhosis. Those with cirrhosis were also more commonly diagnosed under surveillance. NBNC-HCC patients with an absence of cirrhosis were more likely to have a single large tumor, which was more frequently treated with resection (Table 4).

In terms of the mode of detection, $25.5 \%$ of patients 
Table 4. Comparison of characteristics according to the presence of cirrhosis in NBNC-HCC $(n=447)$

\begin{tabular}{|c|c|c|c|}
\hline Variable & Non-cirrhosis $(n=148)$ & Cirrhosis $(n=299)$ & $p$ value \\
\hline Age, yr & $66(58-73)$ & $67(61-73)$ & 0.446 \\
\hline Male sex & $129(87.2)$ & $252(84.3)$ & 0.419 \\
\hline Body mass index ${ }^{\mathrm{a}}, \mathrm{kg} / \mathrm{m}^{2}$ & & & 0.680 \\
\hline$<18.5$ & $4(2.7)$ & $6(2.0)$ & \\
\hline $18.5-22.9$ & $51(34 \cdot 5)$ & $92(30.8)$ & \\
\hline $23.0-24.9$ & $30(20.3)$ & $65(21.7)$ & \\
\hline$\geq 25$ & $62(41.9)$ & $129(43.1)$ & \\
\hline Heavy alcohol use & $79(53.4)$ & $190(63.5)$ & 0.039 \\
\hline Diabetes & $54(36.2)$ & $158(51.6)$ & 0.001 \\
\hline Hypertension & $84(56.8)$ & $162(54.2)$ & 0.606 \\
\hline Dyslipidemia & $23(15.5)$ & $23(7.7)$ & 0.010 \\
\hline Family history of HCC & $14(9.5)$ & $15(5.0)$ & 0.073 \\
\hline Mode of detection & & & $<0.001$ \\
\hline Under surveillance & $7(4.7)$ & $107(35.8)$ & \\
\hline Incidental by screening & $83(56.1)$ & $113(37.8)$ & \\
\hline Symptomatic & $58(39.2)$ & $79(26.4)$ & \\
\hline $\mathrm{HBcIgG}^{\mathrm{b}}$ & & & 0.802 \\
\hline Yes & $76(51.4)$ & $159(53.2)$ & \\
\hline No & $18(12.2)$ & $40(13.4)$ & \\
\hline Child-Pugh class & & & $<0.001$ \\
\hline A & $146(98.6)$ & $243(81.3)$ & \\
\hline B & $2(1.4)$ & $51(17.1)$ & \\
\hline $\mathrm{C}$ & o & $5(1.7)$ & \\
\hline No. of tumors & & & 0.004 \\
\hline Single & $111(75.0)$ & $177(59.2)$ & \\
\hline Multiple & $32(21.6)$ & $107(35.8)$ & \\
\hline Diffuse/infiltrative & $5(3.4)$ & $15(5.0)$ & \\
\hline Tumor size, $\mathrm{cm}$ & & & $<0.001$ \\
\hline$<5$ & $60(40.5)$ & $190(63.5)$ & \\
\hline$\geq 5$ & $88(59.5)$ & $109(36.5)$ & \\
\hline Portal vein thrombosis & $24(16.2)$ & $66(22.1)$ & 0.146 \\
\hline BCLC stage & & & 0.010 \\
\hline o & $8(5.4)$ & $39(13.0)$ & \\
\hline A & $73(49 \cdot 3)$ & $119(39.8)$ & \\
\hline B & $16(10.8)$ & $41(13.7)$ & \\
\hline $\mathrm{C}$ & $51(34.5)$ & $92(30.8)$ & \\
\hline $\mathrm{D}$ & o & $8(2.7)$ & \\
\hline AFP & $10.7(3.7-348.5)$ & $11.3(6.0-226.5)$ & 0.225 \\
\hline PIVKA-II & $185.0(33.0-1,200.0)$ & $94.0(27.0-1,200.0)$ & 0.152 \\
\hline Initial treatment & & & $<0.001$ \\
\hline
\end{tabular}


Table 4. Continued

\begin{tabular}{lcc}
\hline Variable & Non-cirrhosis $(\mathrm{n}=148)$ & Cirrhosis $(\mathrm{n}=299)$ \\
\hline Resection & $64(43.2)$ & $53(17.7)$ \\
RF ablation & $17(11.5)$ & $55(18.4)$ \\
TACE & $43(29.1)$ & $134(44.8)$ \\
Others & $16(10.8)$ & $23(7.7)$ \\
Best supportive care & $8(5.4)$ & $34(11.4)$ \\
\hline
\end{tabular}

Values are presented as median (interquartile range) or number (\%).

NBNC, non-B non-C; HCC, hepatocellular carcinoma; HBcIgG, hepatitis B core immunoglobulin G antibody; BCLC, Barcelona Clinic Liver Cancer; AFP, alpha-fetoprotein; PIVKA-II, prothrombin-induced by vitamin K antagonist-II; RF, radiofrequency; TACE, transarterial chemoembolization.

${ }^{\mathrm{a}}$ Missing in one and seven patients, respectively.

${ }^{\mathrm{b}}$ Missing in $54(36.5 \%)$ and $100(33.4 \%)$ patients, respectively.

(114/447) were diagnosed under HCC surveillance. When compared according to the mode of detection, those who presented with symptoms showed the lowest BMI, the lowest incidence of diabetes, the poorest ECOG, the larger tumor burden with a higher prevalence of portal vein thrombosis, a higher level of tumor markers, and a lower incidence of liver cirrhosis. Those diagnosed under surveillance showed poorer liver function and the highest incidence of liver cirrhosis (Table 5). Comparing the survival curves according to the mode of detection, survival was significantly lower among those who presented with symptoms than those who were diagnosed under surveillance or incidentally $(p<0.001)$ (Supplementary Fig. 1). When the mode of detection was assessed according to potential etiologies, the rate of diagnosis under regular surveillance was the lowest (18.8\%) in patients with NBNC-NA-NO, 80.0\% in patients with NBNC-O, and $27.7 \%$ in patients with NBNC-A (Fig. 4). Further classification of NBNC-A and NBNC-NA-NO patients according to the presence of cirrhosis revealed that those diagnosed under surveillance accounted for a minority (5.1\% for NBNC-A, 2.9\% for NBNC-NA-NO) of the patients (Supplementary Fig. 2).

\section{DISCUSSION}

In this study, we analyzed a total of 447 consecutive NBNC HCC patients (13.4\%) among 3,347 HCC patients registered in our institution. The proportion of NBNC HCC patients was slightly lower than the proportion reported

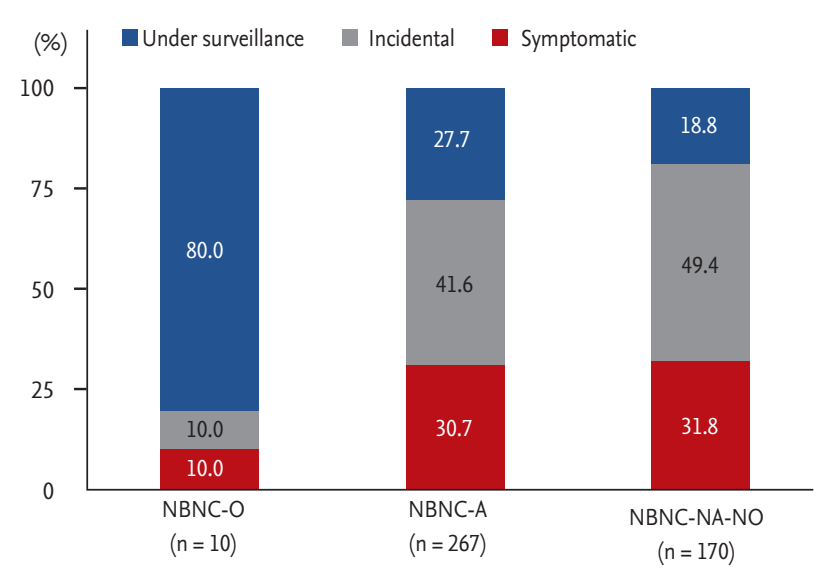

Figure 4. Mode of detection based on potential etiology. NBNC-O, non-B non-C other identifiable cause; NBNC-A, alcohol; NBNC-NA-NO, non-alcohol, no-other identifiable cause.

from other hospitals in Korea, which ranged from $16.1 \%$ to $20.2 \%[5,17]$, and was also lower than that indicated by the nationwide cohort study in Korea, which reported an NBNC HCC prevalence of $27.4 \%$ [3]. In our cohort, the most common potential etiology of NBNC HCC was heavy alcohol use $(n=267,59.7 \%)$. Patients with other identifiable causes comprised only 10 patients $(2.2 \%)$. One hundred and seventy patients showed no history of heavy alcoholism or any other identifiable chronic liver disease. The 5-year survival rate of the cohort was 16.3\% with tumor size, portal vein thrombosis, and initial treatment modality serving as independent risk factors for survival.

In our cohort, 170 patients were classified as NBNC- 
Table 5. Comparison based on mode of detection

\begin{tabular}{|c|c|c|c|c|}
\hline Variable & Under surveillance $(\mathrm{n}=114)$ & Incidental $(n=196)$ & Symptomatic $(n=137)$ & $p$ value \\
\hline Age, yr, median (IQR) & $66(60-72)$ & $68(61-73)$ & $66(56-73)$ & 0.296 \\
\hline Male sex & $90(78.9)$ & $171(87.2)$ & $120(87.6)$ & 0.090 \\
\hline Body mass index & $24.8(22.9-27.1)$ & $24.5(22.0-26.8)$ & $23.5(21.4-25.7)$ & 0.006 \\
\hline Heavy alcohol use & $75(65.8)$ & $111(56.6)$ & $83(60.6)$ & 0.281 \\
\hline Diabetes & $65(57.0)$ & $93(47.4)$ & $53(38.7)$ & 0.015 \\
\hline Hypertension & $58(50.9)$ & $115(58.7)$ & $73(53 \cdot 3)$ & 0.365 \\
\hline Dyslipidemia & $7(6.1)$ & $21(10.7)$ & $18(13.1)$ & 0.186 \\
\hline Family history of HCC & $8(7.0)$ & $11(5.6)$ & $10(7 \cdot 3)$ & 0.799 \\
\hline $\mathrm{HBcIgG}^{\mathrm{b}}$ & & & & 0.294 \\
\hline Yes & $55(48.2)$ & $111(56.6)$ & $69(50.4)$ & \\
\hline No & $12(10.5)$ & $24(12.2)$ & $22(16.1)$ & \\
\hline Liver cirrhosis & $107(93.9)$ & $113(57.7)$ & $79(57.7)$ & $<0.001$ \\
\hline Child-Pugh class & & & & 0.017 \\
\hline A & $90(78.9)$ & $178(90.8)$ & $121(88.3)$ & \\
\hline B & $23(20.2)$ & $15(7.7)$ & $15(10.9)$ & \\
\hline $\mathrm{C}$ & $1(0.9)$ & $3(1.5)$ & $1(0.7)$ & \\
\hline ECOG & & & & 0.040 \\
\hline o & $105(92.1)$ & $180(91.8)$ & $115(83.9)$ & \\
\hline$\geq 1$ & $9(7.9)$ & $16(8.2)$ & $22(16.1)$ & \\
\hline No. of tumors & & & & 0.334 \\
\hline Single & $70(61.4)$ & $129(65.8)$ & $89(65.0)$ & \\
\hline Multiple & $40(35.1)$ & $61(31.1)$ & $38(27.7)$ & \\
\hline Diffuse/infiltrative & $4(3.5)$ & $6(3.1)$ & $10(7.3)$ & \\
\hline Tumor size, $\mathrm{cm}$ & & & & $<0.001$ \\
\hline$<5$ & $99(86.8)$ & $114(58.2)$ & $37(27.0)$ & \\
\hline$\geq 5$ & $15(13.2)$ & $82(41.8)$ & $100(73.0)$ & \\
\hline Portal vein thrombosis & $11(9.6)$ & $31(15.8)$ & $48(35.0)$ & $<0.001$ \\
\hline BCLC stage & & & & $<0.001$ \\
\hline o & $25(21.9)$ & $19(9.7)$ & $3(2.2)$ & \\
\hline A & $52(45.6)$ & $93(47.4)$ & $47(34 \cdot 3)$ & \\
\hline B & $15(13.2)$ & $31(15.8)$ & $11(8.0)$ & \\
\hline $\mathrm{C}$ & $21(18.4)$ & $50(25.5)$ & $72(52.6)$ & \\
\hline $\mathrm{D}$ & $1(0.9)$ & $3(1.5)$ & $4(2.9)$ & \\
\hline AFP & $7.5(4.6-18.5)$ & $10.9(4.8-200.1)$ & $52.9(8.3-7464.3)$ & $<0.001$ \\
\hline PIVKA-II, median (IQR) & $43.0(21.0-142.0)$ & $95.0(30.0-1,013.0)$ & $1,200.0(114.0-1,200.0)$ & $<0.001$ \\
\hline Initial treatment modality & & & & $<0.001$ \\
\hline Resection & $15(13.2)$ & $73(37.2)$ & $29(21.2)$ & \\
\hline RF ablation & $36(31.6)$ & $28(14 \cdot 3)$ & $8(5.8)$ & \\
\hline TACE & $51(44 \cdot 7)$ & $71(36.2)$ & $55(40.1)$ & \\
\hline Others & $5(4.4)$ & $13(6.6)$ & $21(15 \cdot 3)$ & \\
\hline Best supportive care & $7(6.1)$ & $11(5.6)$ & $24(17.5)$ & \\
\hline
\end{tabular}

Values are presented as number (\%) or median (range) unless otherwise indicated.

IQR, interquartile range; HCC, hepatocellular carcinoma; HBcIgG, hepatitis B core immunoglobulin G antibody; ECOG, Eastern Cooperative Oncology Group; BCLC, Barcelona Clinic Liver Cancer; AFP, alpha-fetoprotein; PIVKA-II, prothrombin-induced by vitamin $\mathrm{K}$ antagonist-II; RF, radiofrequency; TACE, transarterial chemoembolization.

${ }^{\mathrm{b}}$ Missing in 47 (41.2\%), 61 (31.1\%) and 46 (33.6\%) patients, respectively. 
NA-NO HCC. Despite missing data for quite a few patients, a majority of the NBNC-NA-NO patients showed positive results for anti-HBc, and many of these carried concomitant metabolic risk factors. Among 115 NBNCNA-NO patients with available HBcIgG results, 75 patients (65.2\%) showed both HBcIgG positivity and had metabolic risk factors, 21 patients $(18.1 \%)$ showed HBcIgG positivity without metabolic risk factors, 16 patients (13.7\%) had metabolic risk factors without showing HBcIgG positivity, and only three patients $(2.5 \%)$ showed $\mathrm{HBcIgG}$ negativity without metabolic risk factors. Consistent with our findings, another study on potential etiology of NBNC HCC in Korea suggested that prior HBV exposure, defined as anti-HBc positivity, could be a potential etiology in NBNC HCC patients [5]. Another Korean study reported that cryptogenic HCC (no HBV, no HCV, and no alcohol) was significantly associated with higher visceral fat, suggesting that these patients may have burnt-out NAFLD-related HCC [6]. NAFLD-related HCC has been well described in Western patients [18,19], and is usually defined as HCC in patients with a histologic evidence of NAFLD, or the presence of metabolic syndrome in the absence of HCV, HBV, or alcoholic liver disease [18]. However, HBsAg-negative patients with prior HBV exposure showed HBV DNA integration [20], which might induce tumor formation associated with occult HBV infection [21]. Korea is an endemic area of HBV $[8,22]$, where occult HBV infection has been reported among patients with isolated anti-HBc [23] and can be an important risk factor for the development of HCC in patients without HBsAg [24]. There can also be a synergistic effect on the development of HCC among patients with prior HBV exposure and concomitant metabolic risk factors [24]. In this study, evidence of steatosis was found either radiologically or histologically in 33 patients. However, it was difficult to classify patients in the NBNC-NA-NO HCC category into occult HBV infection-related or NAFLD-related groups, because quite a bit of patient data were missing for anti-HBc results, many had both anti-HBc and metabolic risk factors, and burnt-out NAFLD-related HCC can be present where liver fat is depleted. Although the precise etiological factors (e.g., non-alcoholic fatty liver or occult HBV infection) and their relative contribution to hepatocarcinogenesis is yet to be determined, our data indicate that NBNC-NA-NO HCC cases are characterized by a high incidence of prior HBV exposure and concomitant metabolic risk factors, resulting in a synergistic effect on hepatocarcinogenesis.

In this study, a substantial number of NBNC HCC patients (33\%) notably showed no evidence of cirrhosis based on histological, radiological, endoscopic, and serologic results. Another cohort study from Korea also reported the absence of cirrhosis in $27.5 \%$ of NBNC HCC patients [5], with a cirrhosis rate comparable to that of our study. Several studies have reported that fibrosis and cirrhosis are not always necessary for the development of HCC, especially for NAFLD-related HCC [17,18,25,26]. Kim et al. [27] compared histologic findings between HBV-related HCC and NBNC HCC using data of surgically resected HCC patients in Korea and reported that most HBV-related HCC patients had septal fibrosis (85.6\%, 297/360 patients) but were non-cirrhotic, while more than half (52.7\%, 49/103 patients) of non-cirrhotic NBNC HCC patients had no fibrosis. In our study, the proportion of non-cirrhotic HCC patients was the lowest among NBNC-O HCC (10\%) and the highest among NBNC-NA-NO HCC (40\%). Thus, it is evident that HCC can develop in non-cirrhotic liver, which comprises a significant proportion of NBNC-NA-NO HCC cases.

In our cohort, only $25 \%$ of NBNC HCC patients were diagnosed under surveillance. The proportion diagnosed under surveillance was the lowest in the NBNCNA-NO group (19\%) where the proportion of cirrhosis was the lowest (40\%), and was the highest in the NBNC-O group (80\%) where the proportion of cirrhosis was the highest (90\%). Among patients without cirrhosis, those diagnosed under surveillance comprised a minority (5.1\% of non-cirrhotic NBNC-A patients, $2.9 \%$ of non-cirrhotic NBNC-NA-NO patients). Similarly, $93.9 \%$ of the 114 patients diagnosed under surveillance had cirrhosis, while the cirrhosis rate was $57.7 \%$ among those diagnosed incidentally and symptomatically. In this study, we observed that those diagnosed under surveillance had better survival than symptomatic patients. Futher studies are needed that can provide the best strategies for identifying the at-risk population among NBNC patients who may benefit from HCC surveillance.

In this study, we observed a few unique characteristics of NBNC-NA-NO patients. When compared with NBNC-A patients, the age at diagnosis was significantly older in NBNC-NA-NO patients. Moreover, 80.6\% 
of NBNC-NA-NO patients were aged 60 years or older and more than half (48.2\%) were aged 70 and above. A majority of tumors were single (73.5\%) and nearly half (48.8\%) were larger than $5 \mathrm{~cm}$. Lee et al. [6], also suggested that cryptogenic HCC is associated with an older age at diagnosis and a higher prevalence of single nodular presentation than HCC originating in other chronic liver diseases. Adam et al. [28] reported that hepatocarcinogenesis in a non-cirrhotic liver was associated with a reduced number of clonal hepatocellular patches, analogous to aging-related changes, which may explain the older age at diagnosis in the absence of cirrhosis in many NBNC-NA-NO patients. Another study from Korea also reported that patients with cryptogenic HCC were much older than those with HCC associated with other etiologies [17]. However, the outcome of NBNCNA-NO HCC was similar to NBNC-A HCC, despite several differences in baseline characteristics.

There are some limitations of this study. First, as this was a retrospective study, some key variables (e.g., anti-HBc) were missing in a substantial number of patients. Misclassification bias may also be present, as histologic, radiologic, and endoscopic data used to define cirrhosis were based on existing electronic medical records developed by many doctors. Another limitation is possible inter-observer and intra-observer variation in defining cirrhosis histologically and radiologically, as well as in defining varices endoscopically, which may result in the over- or under-estimation of cirrhosis incidence in our population. However, since the doctors who evaluated the histologic, radiologic and endoscopic findings were unaware of the aim of this study, this type of bias is non-differential. Second, although we consecutively enrolled patients so as to minimize selection bias, this study was confined to a single referral center, and thus selection bias cannot be ruled out. Third, as discussed, NAFLD-related HCC could not be accurately assessed in this study.

In summary, we systematically analyzed consecutive NBNC HCC patients in a single center. The potential etiological factors of NBNC HCC patients included NBNC-A and NBNC-O, however, many patients were classified as NBNC-NA-NO. NBNC-NA-NO patients were characterized by a high incidence of anti-HBc and a high incidence of concomitant metabolic risk factors. Notably, $40 \%$ of NBNC-NA-NO patients showed no evi- dence of cirrhosis based on histology, radiology, endoscopy, and FIB-4 scores, and only $18.8 \%$ were diagnosed under HCC surveillance. These findings imply that prior HBV infection and metabolic risk factors may be major contributing factors in the development of HCC in NBNC-NA-NO patients in Korea, even in the absence of cirrhosis.

\section{KEY MESSAGE}

1. Heavy alcohol use or any other specific cause was not found in $38.0 \%$ of non-B non-C hepatocellular carcinoma (NBNC HCC) patients.

2. These NBNC-NA-NO (non-alcohol no-other identifiable cause) HCC group showed the highest proportion of non-cirrhotic patients (40.0\%) and the lowest proportion of diagnosis under regular surveillance (18.8\%).

3. Prior hepatitis B virus infection and metabolic risk factors may be major causes of HCC in NBNC-NA-NO patients.

\section{Conflict of interest}

No potential conflict of interest relevant to this article was reported.

\section{REFERENCES}

1. Jung KW, Won YJ, Oh CM, Kong HJ, Lee DH, Lee KH. Prediction of cancer incidence and mortality in Korea, 2017. Cancer Res Treat 2017;49:306-312.

2. Ferlay J, Soerjomataram I, Dikshit R, et al. GLOBOCAN 2012 v1.o, Cancer incidence and mortality worldwide: IARC CancerBase No. 11 [Internet]. Lyon (FR): International Agency for Research on Cancer; c2018 [cited 2018 Nov 26]. Available from: http://globocan.iarc.fr.

3. Kim BH, Lim YS, Kim EY, et al. Temporal improvement in survival of patients with hepatocellular carcinoma in a hepatitis B virus-endemic population. J Gastroenterol Hepatol 2018;33:475-483.

4. Cho EJ, Kwack MS, Jang ES, et al. Relative etiological role of prior hepatitis B virus infection and nonalcoholic fatty liver disease in the development of non-B non-C hepatocellular carcinoma in a hepatitis B-endemic area. Diges- 
tion 2011;84 Suppl 1:17-22.

5. Lee SB, Kim KM, An J, et al. Clinical characteristics and potential aetiologies of non-B non-C hepatocellular carcinoma in hepatitis B virus endemic area. Liver Int 2016;36:1351-1361.

6. Lee SS, Jeong SH, Byoun YS, et al. Clinical features and outcome of cryptogenic hepatocellular carcinoma compared to those of viral and alcoholic hepatocellular carcinoma. BMC Cancer 2013;13:335.

7. Korean Association for the Study of the Liver (KASL). KASL clinical practice guidelines: management of nonalcoholic fatty liver disease. Clin Mol Hepatol 2013;19:325348.

8. Cho EJ, Kim SE, Suk KT, et al. Current status and strategies for hepatitis B control in Korea. Clin Mol Hepatol 2017;23:205-211.

9. Korean Liver Cancer Study Group and National Cancer Center, Korea. Practice guidelines for management of hepatocellular carcinoma 2009. Korean J Hepatol 2009;15:391-423.

10. Korean Liver Cancer Study Group (KLCSG); National Cancer Center, Korea (NCC). 2014 KLCSG-NCC Korea practice guideline for the management of hepatocellular carcinoma. Gut Liver 2015;9:267-317.

11. Sterling RK, Lissen E, Clumeck N,et al. Development of a simple noninvasive index to predict significant fibrosis in patients with HIV/HCV coinfection. Hepatology 2006;43:1317-1325.

12. Kim BK, Kim DY, Park JY, et al. Validation of FIB-4 and comparison with other simple noninvasive indices for predicting liver fibrosis and cirrhosis in hepatitis B virus-infected patients. Liver Int 2010;30:546-553.

13. O'Shea RS, Dasarathy S, McCullough AJ. Alcoholic liver disease. Am J Gastroenterol 2010;105:14-32.

14. Chalasani N, Younossi Z, Lavine JE, et al. The diagnosis and management of non-alcoholic fatty liver disease: practice guideline by the American Association for the Study of Liver Diseases, American College of Gastroenterology, and the American Gastroenterological Association. Hepatology 2012;55:2005-2023.

15. Allen JP, Wilson VB. Assessing Alcohol Problems: A Guide for Clinicians and Researchers. 2nd ed. Bethesda (MD): U.S. Dept. of Health and Human Services, Public Health Service, National Institutes of Health, National Institute on Alcohol Abuse and Alcoholism, 2003.
16. American Diabetes Association. 1. Promoting health and reducing disparities in populations. Diabetes Care 2017;40(Suppl 1):S6-S10.

17. Kwak HW, Park JW, Koh YH, Lee JH, Yu A, Nam BH. Clinical characteristics of patients with cryptogenic hepatocellular carcinoma in a hepatitis B virus-endemic area. Liver Cancer 2016;5:21-36.

18. Mittal S, Sada YH, El-Serag HB, et al. Temporal trends of nonalcoholic fatty liver disease-related hepatocellular carcinoma in the veteran affairs population. Clin Gastroenterol Hepatol 2015;13:594-601.

19. Marrero JA, Fontana RJ, Su GL, Conjeevaram HS, Emick DM, Lok AS. NAFLD may be a common underlying liver disease in patients with hepatocellular carcinoma in the United States. Hepatology 2002;36:1349-1354.

20. Saitta C, Tripodi G, Barbera A, et al. Hepatitis B virus (HBV) DNA integration in patients with occult HBV infection and hepatocellular carcinoma. Liver Int 2015;35:2311-2317.

21. Pollicino T, Squadrito G, Cerenzia G, et al. Hepatitis B virus maintains its pro-oncogenic properties in the case of occult HBV infection. Gastroenterology 2004;126:102-110.

22. Sinn DH, Cho EJ, Kim JH, Kim DY, Kim YJ, Choi MS. Current status and strategies for viral hepatitis control in Korea. Clin Mol Hepatol 2017;23:189-195.

23. Kang SY, Kim MH, Lee WI. Occult hepatitis B virus infection in Korean patients with isolated anti-HBc. Arch Virol 2014;159:227-233.

24. Lee JJ, Kwon OS. Occult hepatitis B virus infection and hepatocellular carcinoma. Korean J Gastroenterol 2013;62:160164.

25. Noureddin M, Rinella ME. Nonalcoholic fatty liver disease, diabetes, obesity, and hepatocellular carcinoma. Clin Liver Dis 2015;19:361-379.

26. Michelotti GA, Machado MV, Diehl AM. NAFLD, NASH and liver cancer. Nat Rev Gastroenterol Hepatol 2013;10:656-665.

27. Kim JM, Kwon CH, Joh JW, et al. Outcomes after curative hepatectomy in patients with non-B non-C hepatocellular carcinoma and hepatitis B virus hepatocellular carcinoma from non-cirrhotic liver. J Surg Oncol 2014;110:976981.

28. Adam AC, Faudou V, Paschen SA, et al. Hepatocarcinogenesis in non-cirrhotic liver is associated with a reduced number of clonal hepatocellular patches in non-tumorous liver parenchyma. J Pathol 2012;228:333-340. 
Supplementary Table 1. Comparison of patients with NBNC-NA-NO according to HBcIgG positivity

\begin{tabular}{|c|c|c|c|}
\hline Characteristic & $\operatorname{HBcIgG}(+)(n=96,89.7 \%)$ & $\operatorname{HBcIgG}(-)(\mathrm{n}=19,10.3 \%)$ & $p$ value \\
\hline Age, yr & $67(60-74)$ & $72(58-77)$ & 0.407 \\
\hline Male sex & $69(71.9)$ & $12(63.2)$ & 0.447 \\
\hline Body mass index & $24.5(17.8-32.7)$ & $23.4(16.8-33.5)$ & 0.519 \\
\hline Diabetes & $46(47 \cdot 9)$ & $10(52.6)$ & 0.707 \\
\hline Hypertension & $56(58.3)$ & $12(63.2)$ & 0.696 \\
\hline Dyslipidemia & $14(14.6)$ & $3(15.8)$ & 0.892 \\
\hline Family history of HCC & $11(11.5)$ & $3(15.8)$ & 0.700 \\
\hline Mode of detection & & & 0.024 \\
\hline Under surveillance & $18(18.8)$ & $2(10.5)$ & \\
\hline Incidental & $54(56.3)$ & $6(31.6)$ & \\
\hline Symptomatic & $24(25 \cdot 0)$ & $11(57 \cdot 9)$ & \\
\hline Liver cirrhosis & $58(60.4)$ & $14(73.7)$ & 0.275 \\
\hline Child-Pugh class & & & 0.598 \\
\hline A & $85(88.5)$ & $16(84.2)$ & \\
\hline $\mathrm{B}$ & $9(9.4)$ & $3(15.8)$ & \\
\hline $\mathrm{C}$ & $2(2.1)$ & 0 & \\
\hline ECOG & & & 0.300 \\
\hline 0 & $84(87.5)$ & $15(78.9)$ & \\
\hline$\geq 1$ & $12(12.5)$ & $4(21.1)$ & \\
\hline No. of tumors & & & 0.560 \\
\hline Single & $68(70.8)$ & $16(84.2)$ & \\
\hline Multiple & $23(24.0)$ & $3(15.8)$ & \\
\hline Diffuse/infiltrative & $5(5.2)$ & 0 & \\
\hline Tumor size, $\mathrm{cm}$ & & & 0.258 \\
\hline$<5$ & $49(51.0)$ & $7(36.8)$ & \\
\hline$\geq 5$ & $47(49.0)$ & $12(63.2)$ & \\
\hline Portal vein thrombosis & $22(22.9)$ & $5(26.3)$ & 0.749 \\
\hline $\log _{10}$ AFP & $1.08(0.65-3.10)$ & $1.17(0.71-2.77)$ & 0.949 \\
\hline PIVKA-II & $121.0(26.7-1,200.0)$ & $1,200.0(27.0-6,866.0)$ & 0.306 \\
\hline Initial treatment modality & & & 0.409 \\
\hline Resection, RF ablation & $44(45.8)$ & $6(31.6)$ & \\
\hline TACE, others & $43(44.8)$ & $10(52.6)$ & \\
\hline Best supportive care & $9(9.4)$ & $3(15.8)$ & \\
\hline
\end{tabular}

Values are presented as number (\%) or median (interquartile range). Fifty-five patients without HBcIgG were excluded from this table.

NBNC-NA-NO, non-B non-C non-alcohol, no-other identifiable cause; HBcIgG, hepatitis B core immunoglobulin G antibody; HCC, hepatocellular carcinoma; ECOG, Eastern Cooperative Oncology Group; AFP, alpha-fetoprotein; PIVKA-II, prothrombin-induced by vitamin K antagonist-II; RF, radiofrequency; TACE, transarterial chemoembolization. 


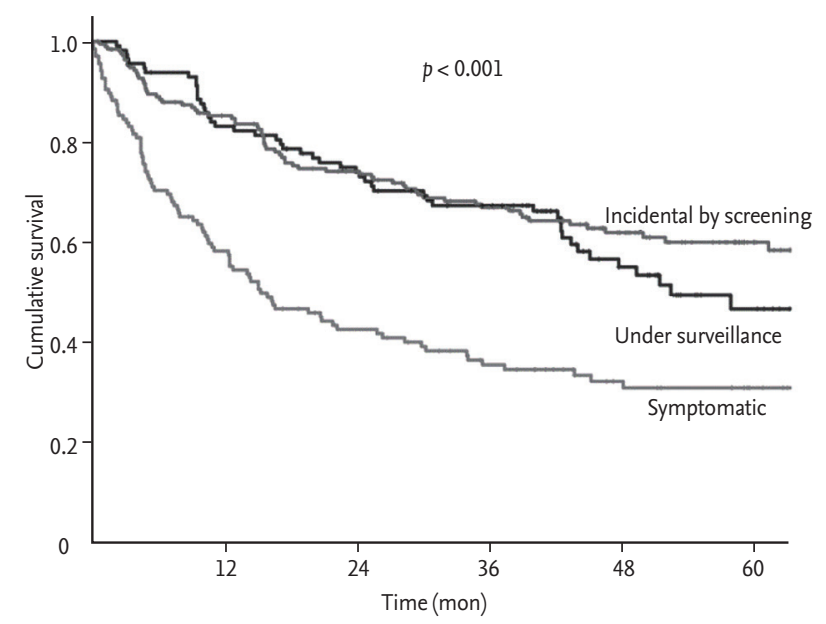

Supplementary Figure 1. Mode of detection and survival. 


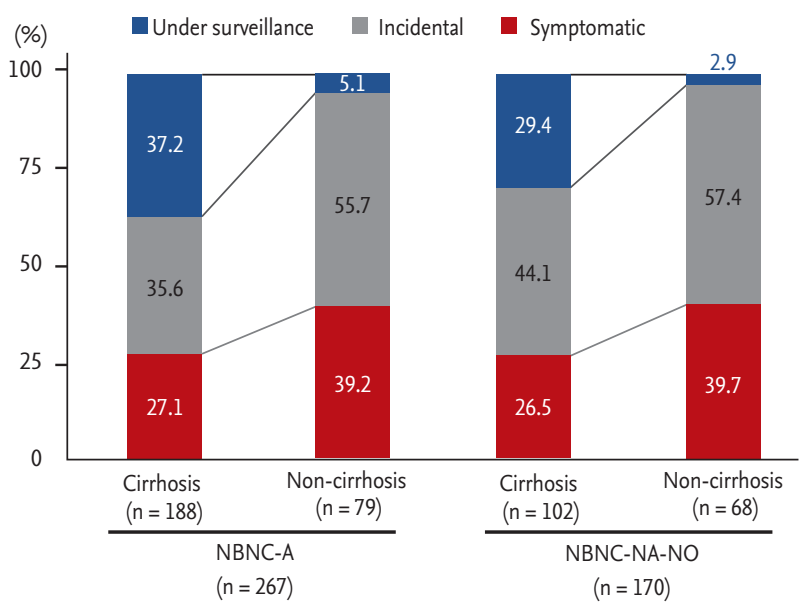

Supplementary Figure 2. Mode of detection based on potential etiology and presence of cirrhosis. NBNC-A, non-B non-C alcohol; NBNC-NA-NO, non-alcohol, no-other identifiable cause. 